\title{
A tensor decomposition-based integrated analysis applicable to multiple gene expression profiles without sample matching
}

Taguchi Y-h. ( $\nabla$ tag@granular.com )

Chuo University

\section{Turki Turki}

King Abdulaziz University

\section{Article}

Keywords: tensor decomposition-based integrated analysis, multiple gene expression profiles

Posted Date: November 4th, 2021

DOI: https://doi.org/10.21203/rs.3.rs-766884/v2

License: (c) (i) This work is licensed under a Creative Commons Attribution 4.0 International License.

Read Full License 


\title{
A tensor decomposition-based integrated analysis applicable to multiple gene expression profiles without sample matching
}

\author{
Y-h. Taguchi ${ }^{1, *}$ and Turki Turki ${ }^{2}$ \\ ${ }^{1}$ Department of Physics, Chuo University, Tokyo, 112-8551 Japan \\ ${ }^{2}$ Department of Computer Science, King Abdulaziz University, Jeddah, 21589, Saudi Arabia \\ *tag@granular.com
}

\begin{abstract}
The integrated analysis of multiple gene expression profiles measured in distinct studies is always problematic because missing sample matches and common labels prevent their integration in fully data-driven, unsupervised training. In this study, we propose a strategy to enable the integration of multiple gene expression profiles among multiple independent studies with neither labeling nor sample matching using tensor decomposition unsupervised feature extraction. We apply this strategy to Alzheimer's disease (AD)-related gene expression profiles that lack precise correspondence among samples, including AD single-cell RNA sequence (scRNA-seq) data. We find that we can select biologically reasonable genes using the integrated analysis. Overall, integrated gene expression profiles can function analogously to prior- and/or transfer-learning strategies in other machine-learning applications. For scRNA-seq, the proposed approach drastically reduces the required computational memory.
\end{abstract}

\section{Introduction}

The integrated analysis of gene expression profiles is generally difficult to accomplish ${ }^{1-4}$. The primary purpose of this task is to compensate for small sample sizes by integrating gene expression profiles measured from multiple studies because the process is generally not straightforward, and matching samples between them are rare. If sample-matching information is missing, and samples are not associated with common labeling (e.g., healthy controls and patients), the requirements of this process are not always fulfilled, even if we simply group gene expression profiles using their labels within individual sets. Hence, the establishment of general frameworks for integrating multiple gene expression profiles without sample matching and common sample labeling would be of considerable benefit. Owing to these basic requirements, such methods must be unsupervised, because the integration of multiple gene expression profiles that share nothing other than the genes between them is impossible. In this study, we employ tensor decomposition (TD) for this purpose ${ }^{5}$.

\section{Results}

Figure S1 shows the workflow of analyses performed in this study.

\section{Integrated Analysis of Datasets 1, 2, and 3}

First, to test whether this simple idea can integrate multiple gene expression profiles lacking sample matching, we attempted to integrate datasets 1,2, and 3. Datasets 1 and 3 comprised cell lines modeled as either healthy controls or ADs, whereas dataset 2 comprised cell lines aiming to mutate AD-associated genes indirectly related to AD-only. After obtaining a singular value vector, $u_{\ell_{1}}$, as described in Eq. (8), we first investigated whether they were associated with classifications. Singular value vectors attributed to the $j_{k}$ th sample, $v_{\ell_{1} j_{k} k}, 1 \leq k \leq 3$, were computed from $u_{\ell_{1} i}$ using Eq. (10), and the coincidence of $v_{\ell_{1} j_{k} k}$ with the classifications of datasets 1, 2, or 3 was investigated (Table S1 and Fig. S2).

It is obvious that $v_{\ell_{1} j_{k} k}, 1 \leq \ell_{1} \leq 5$ were significantly correlated with classifications in any of datasets 1,2 , or 3 . Next, we tried to find $u_{\ell_{1} i}$, which is used for gene selection. Then, we selected $u_{\ell_{1} i}, 1 \leq \ell_{1} \leq 5$ (see Table S5). $P_{i}$ are attributed to $i$ using Eq. (9) while taking $\Omega_{\ell_{1}}=\left\{\ell_{1} \mid 1 \leq \ell_{1} \leq 5\right\}$. The obtained $P_{i}$ were corrected using the BH criterion ${ }^{5}$, and $565 i$ (genes) associated with adjusted $P_{i}$ less than 0.01 were selected. To validate the selected 565 genes biologically, gene symbols were uploaded to Enrichr ${ }^{6}$. We observed various enrichments (Data S1). For example, the "KEGG 2021 Human" category listed six neurodegenerative disease-related pathways within the top-10 pathways (Table S2(A)). Similarly, the "Jensen Diseases" category listed four neurodegenerative diseases within the top-10(see Table S2(A)). Additionally, "Disease Perturbations from 
GEO down" and "Disease Perturbations from GEO up" listed similar neurodegenerative diseases within the top-ranked diseases (Data S1). We noted the "Allen Brain Atlas up" and "Allen Brain Atlas down" categories in which many AD-related brain regions were highly ranked (Data S1). Enrichment analyses using databases other than Enrichr also reported convincing results. The top- and the third-ranked terms in the "GAD_DISEASE_CLASS" category of DAVID" ${ }^{7,8}$ were PSYCH and NEUROLOGICAL, respectively (Table S2(A)). The top-10 ranked terms in the "GAD_DISEASE" category of DAVID included five neurodegenerative diseases (Table S2(A)). g:Profiler ${ }^{9}$ listed 16 KEGG pathways as significantly enriched with gene symbols associated with 565 selected genes; 16 pathways included six neurodegenerative disease-related pathways (Table S2(A)). Thus, obviously TD-based unsupervised FE was used to integrate three gene expression profiles lacking sample matching, genes of expressions associated with classifications in three independent studies were identified, and genes selected were enriched by various neurodegenerative disease-related terms from three biological databases. This suggests that TD-based unsupervised FE is a promising method for integrating gene expression profiles without sample matching.

\section{Drug Repositioning using the Tensor Obtained with Datasets 1, 2, and 3}

Previously in Ref. ${ }^{10}$, we demonstrated that integrated analyses of gene expression profiles between model animals treated by various drugs and patients were useful to determining which drug compounds are effective against diseases. There, we integrated only one disease gene expression profile with that of the drug treatment. In the present study, we integrated more gene expression profiles of diseases with those of drug treatment because of the novel framework introduced herein. We employed the gene expression profile (i.e., the integration of datasets 1,2 , and $3, x_{i \ell k}, 1 \leq k \leq 3$ ), and we employed dataset 4 as a gene expression profile of drug treatment. We obtained $x_{i \ell 4}$, as described in Materials and Methods, to be integrated with $x_{i \ell k}, 1 \leq k \leq 3$, as obtained previously. HOSVD was applied to the obtained $x_{i \ell k}, 1 \leq k \leq 4$.

First, we sought to validate the singular value vectors, $v_{\ell_{1} j_{k} k}$, associated with classifications (Table S1 and Fig. S3). It is obvious that they are coincident with the classifications of individual datasets. Then, we selected $u_{\ell_{1}}, 1 \leq \ell_{1} \leq 5$ as those associated with $u_{\ell_{2} \ell}, 1 \leq \ell_{2} \leq 4$ by investigating $G\left(\ell_{1}, \ell_{2}, \ell_{3}\right)$ in Eq. (8) while fixing $1 \leq \ell_{2} \leq 4$ (see Table $S 6$ ). $P_{i}$ are attributed to $i$ using Eq. (9) while taking $\Omega_{\ell_{1}}=\left\{\ell_{1} \mid 1 \leq \ell_{1} \leq 5\right\}$. The obtained $P_{i}$ were corrected using the $\mathrm{BH}$ criterion and $544 i$ genes associated with adjusted $P_{i}$ less than 0.01 , as selected. To validate the selected 544 genes biologically, their associated gene symbols were uploaded to Enrichr. We then observed various enrichments (Data S2). For example, the "KEGG 2021 Human" category listed six neurodegenerative disease pathways within the top-10 pathways (Table S2(B)). Similarly, the "Jensen Diseases" category listed four neurodegenerative diseases within the top-10 diseases as well (Table S2(B)). In addition, "Disease Perturbations from GEO down" and "Disease Perturbations from GEO up" listed similar neurodegenerative diseases within the top-ranked diseases (Data S2). Examining the "Allen Brain Atlas up" and "Allen Brain Atlas down" categories, many AD-related brain regions were highly ranked (Data S2). Enrichment analyses using databases other than Enrichr also reported convincing results. The first- and fourth-ranked terms in the "GAD_DISEASE_CLASS" category of DAVID were PSYCH and NEUROLOGICAL, respectively (Table S2(B)). The top-10 ranked terms in the "GAD_DISEASE" category of DAVID included four neurodegenerative diseases (Table S2(B)). The g:Profiler listed 13 KEGG pathways as significantly enriched with gene symbols associated with 544 selected genes; 13 pathways included six neurodegenerative disease-related pathways (Table S2(B)). Hence, it is clear that the TD-based unsupervised FE integrated four gene expression profiles lacking sample matching; it identified genes whose expressions were associated with classifications in three independent studies simultaneously; and it selected genes that were enriched by various neurodegenerative disease-related terms from three biological databases.

Next, we tried to identify how this strategy ranked drugs based upon gene expression profiles in dataset 4 . After computing $v_{\ell_{1} j_{4}^{[1]} j_{4}^{[2]} j_{4}^{[3]} \in} \in \mathbb{R}^{60617 \times 94 \times 4 \times 3}$ by Eq. (11), HOSVD was applied to $v_{\ell_{1} j_{4}^{[1]} j_{4}^{[2]} j_{4}^{[3]}}$ with fixed $\ell_{1}$, and we obtained

$$
v_{\ell_{1} j_{4}^{[1]} j_{4}^{[2]} j_{4}^{[3]}}=\sum_{\tilde{\ell}_{1}=1}^{94} \sum_{\tilde{\ell}_{2}=1}^{4} \sum_{\tilde{\ell}_{3}=1}^{3} \tilde{G}^{\left[\ell_{1}\right]}\left(\tilde{\ell}_{1}, \tilde{\ell}_{2}, \tilde{\ell}_{3}\right) \tilde{u}_{\tilde{\ell}_{1} j_{4}^{[1]}}^{\left[\ell_{1}\right]} \tilde{u}_{\tilde{\ell}_{2} j_{4}^{[2]}}^{\left[\ell_{1}\right]} \tilde{u}_{\tilde{\ell}_{3} j_{4}^{[3]}}^{\left[\ell_{1}\right]},
$$

where $\tilde{u}_{\tilde{\ell}_{1} j_{4}^{[1]}}^{\left[\ell_{1}\right]} \in \mathbb{R}^{94 \times 94}$ was a singular value vector attributed to drugs, $\tilde{u}_{\tilde{\ell}_{2} j_{4}^{[2]}}^{\left[\ell_{1}\right]} \in \mathbb{R}^{4 \times 4}$ was a singular value vector attributed to dose density, and $\tilde{u}_{\tilde{\ell}_{3} j_{4}^{[3]}}^{\left[\ell_{1}\right]} \in \mathbb{R}^{3 \times 3}$ was a singular value vector attributed to biological replicates. We checked the drugs having $\operatorname{larger} \sum_{\tilde{\ell}_{1}=1}^{5}\left(\tilde{u}_{\tilde{\ell}_{1} j_{4}^{[1]}}^{\left[\ell_{1}\right]}\right)^{2}$, and Table S3 shows the top-five drugs for $1 \leq \ell_{1} \leq 4$. Because most were regarded as effective in the original study ${ }^{11}$, drug repositioning seems to have been successful.

\section{TL using the Tensor Obtained with Datasets 1, 2, and 3}

One possible application of the integrated analysis of multiple gene expressions lacking sample matching is analogous to $\mathrm{TL}^{12}$, where pre-trained machine-learning models are used to achieve better performance, even with smaller sample sizes. 
Although deep learning (DL) is often used for TL, DL architectures are not suitable for gene expression profile processing. For example, Yifei et $\mathrm{al}^{13}$ found that DL achieved better performance than linear regression (LR) only by less than $7 \%$. This can be attributed to the lack of structure for gene expression profiles. In contrast to images and sentences (or documents) to which DL was applied successfully, gene expressions are orderless vectors of real numbers. In contrast, TD was successfully applied to gene expression profiles ${ }^{5}$ because tensor similarly does not consider the order of real numbers at all. Thus, TD is a suitable architecture for processing gene expression profiles.

To determine whether the integrated analysis of multiple gene expression profiles obtained using datasets 1,2 , and 3 can be used as a pre-trained system in TL, we combined dataset 5 with $x_{i \ell k}, 1 \leq k \leq 3$ as a pre-trained system. Dataset 5 comprises gene expression profiles created by the overexpression of the $\mathrm{ABCC} 1$ gene, which was recently recognized as an AD therapy target ${ }^{14}$. Usually, only gene expression profiles caused by overexpression of $\mathrm{ABCC} 1$ gene are analyzed, and genes whose expression are altered by ABCC1 over-expression are selected. Then, selected genes are compared to those whose expressions are known to have been altered by AD. Nevertheless, this procedure is somewhat indirect because no sample matching between gene expression profiles of $\mathrm{AD}$ and $\mathrm{ABCC} 1$ overexpression experiments exist. In our strategy, because we can directly compare these expression profiles, our strategy is more suitable for the comparison of gene expression profiles of $\mathrm{AD}$ and $\mathrm{ABCC} 1$ over-expressions.

After obtaining the TD as in Eq. (8) for dataset 5, $v_{\ell_{1} j_{5}^{[1]} j_{5}^{[2]} j_{5}^{[3]} 5}$ was computed by Eq. (11); the correlation between six categories in dataset 5 and $v_{\ell_{1} j_{5}^{[1]} j_{5}^{[2]} j_{5}^{[3]} 5}$ was then computed (Table S1), and the results are shown in Fig. S4. Although we have considered scenarios up to $\ell_{1}=5, v_{\ell_{1} j_{5}^{[1]} j_{5}^{[2]} j_{5}^{[3]} 5}$ were coincident with classification. Thus, as expected, the data show correlations between gene expression profiles of $\mathrm{AD}$ and $\mathrm{ABCC} 1$ overexpression through $\mathrm{TD}$.

We selected $u_{\ell_{1}}, 1 \leq \ell_{1} \leq 5$ by investigating $G\left(\ell_{1}, \ell_{2}, \ell_{3}\right)$ in Eq. (8) and fixing $\ell_{2} \in\{1,3,4,5\}$ (see Table S7). $P_{i}$ were attributed to $i$ using Eq. (9) while taking $\Omega_{\ell_{1}}=\left\{\ell_{1} \mid 1 \leq \ell_{1} \leq 5\right\}$. The obtained $P_{i}$ were corrected with the BH criterion and 660 $i$ (genes) associated with adjusted $P_{i}$ less than 0.01 that were selected. To validate the selected 660 genes biologically, gene symbols associated with genes were uploaded to Enrichr, and we observed various enrichments thereof (Data S3). For example, the "KEGG 2021 Human" category listed six neurodegenerative disease-related (Table S2(C)) pathways within the top-10. Similarly, the "Jensen Diseases" category listed four neurodegenerative diseases (Table S2(C)) within the top-10. "Disease Perturbations from GEO down" and "Disease Perturbations from GEO down" listed similar neurodegenerative diseases within the top-ranked diseases (Data S3). In "Allen Brain Atlas up" and "Allen Brain Atlas down" categories, many AD-related brain regions were highly ranked (Data S3). Enrichment analyses using databases other than Enrichr also reported convincing results. The top-three terms in the "GAD_DISEASE_CLASS" category of DAVID were PSYCH, NEUROLOGICAL, and AGING (Table S2(C)). The top-10 terms in the "GAD_DISEASE" category of DAVID included four neurodegenerative diseases (Table S2(C)). The g:Profiler listed 16 KEGG pathways as significantly enriched with gene symbols associated with 633 selected genes; 16 pathways included six neurodegenerative disease-related pathways (Table S2(C)). Thus, we successfully selected genes whose expressions were simultaneously altered by both $\mathrm{AD}$ and $\mathrm{ABCC} 1$ over-expressions and biological properties evaluated by enrichment analysis.

\section{Integrated Analysis of scRNA-seq Data}

In this subsection, we applied our strategy to the integrated analysis of the scRNA-seq dataset. Because individual experiments with scRNA-seq included $\sim 10^{4}$ cells, it was unclear how to integrate multiple scRNA-seq profiles. After obtaining the TD, as shown in Eq. (8), where $k$ was replaced with an integer, $1 \leq c \leq 25$, representing the $c$ th RNA-seq measurement described in $\S$, we focused on $u_{\ell_{3} c} \in \mathbb{R}^{25 \times 25}$. Variable $c$ was divided into four groups, including either AD or healthy controls in either hippocampus or cortex brain regions. Next, we determined which $u_{\ell_{3} c}$ was associated with these four classes (Fig. S5 ). Then, we found that only $u_{6 c}$ was associated with these four classes; categorical regression was applied to $u_{\ell_{3} c}$ for each $\ell_{3}$ separately, and $P$-values were computed and corrected by BH criterion. Only $\ell_{3}=6$ was associated with adjusted $P$-values less than 0.05 . As a result, we found that $\sum_{\ell_{2}} G\left(6 \ell_{2} 6\right)^{2}$ was the largest among $\sum_{\ell_{2}} G\left(\ell_{1} \ell_{2} 6\right)^{2}$, where a summation was taken over $\ell_{2}$ because there was no reason to select one specific $\ell_{2}$ (Table S8). $P_{i}$ were attributed to $i$ using Eq. (9) with $\Omega_{\ell_{1}}=\left\{\ell_{1} \mid 6\right\}$. $P_{i}$ were3 corrected, and $177 i$ (genes) were associated with an adjusted $P_{i}$ of less than 0.01 . Gene symbols associated with these 177 $i$ were uploaded to Enrichr (Data S4). The results were very distinct from those in Table S2. No neurodegenerative disease terms were detected within the top-10 ranked terms in either "KEGG 2021 HUMAN" or "JENSEN DISEASES" categories. Instead, the top-10 ranked terms in the "Human Gene Atlas" category contained several brain tissues (Table S4). In contrast, the "Disease Perturbations from GEO down" and "Disease Perturbations from GEO down" categories included many diseases related to the brain (Table $\mathrm{S} 4$ ).

Although these are only a few examples, notwithstanding the simplicity of our strategy, it successfully listed genes associated with tissue specificity as well as AD. Thus, it is obvious that our proposed strategy can deal with scRNA-seq very easily, even without directly considering the massive number of cells in individual scRNA-seq measurements because we consider only the top-10 singular value vectors within individual scRNA-seq gene expression profiles, including up to $10^{4}$ cells. 


\section{Discussion}

\section{Advantages of the Proposed Implementation}

It is evident that our implementation has at least two unique advantages. The first is that it enables the integration of multiple gene expression profiles without sample matching. Datasets 1, 2, and 3 had sample sizes of 9, 23, and 8 , respectively. Replacing these numbers of samples with those of singular value vectors (i.e., sample size of eight), we obtained a single tensor, $x_{i \ell k}$, to which the HOSVD was easily applied. It is also possible to evaluate the consistency of singular value vectors, $v_{\ell_{1} j_{k} k}$, attributed to samples in individual datasets by computing it from $u_{\ell_{1} i}$ with Eq. (10) after applying HOSVD to $x_{i \ell k}$ with classifications of individual datasets. Because this strategy is clearly applicable to the general number of samples in individual gene expression profiles, it is very useful. Its second advantage is a reduction in the computational memory required for analysis. When we applied this implementation to scRNA-seq data, we employed only the highest-ranked 10 singular value vectors computed by applying SVD to individual scRNA-seq data, rather than considering as many as $\sim 10^{4}$ cells. This drastically reduced the required memory by a factor of 100. Nevertheless, we successfully selected genes associated with various significantly enriched biological terms related to tissue specificity as well as diseases, as expected. Because more cells are expected to be sequenced in individual experiments via scRNA-seq in the future, our strategy is recommended for a wide range of scRNA-seq analysis applications.

\section{Visualization of Relation between Samples without Sample Matching}

Figures S2, S3, and S4 show that the proposed strategy can relate gene expression profiles lacking sample matching with one another. For example, $v_{1 j_{k} k}$ are always coincident with classifications regardless of $k$ or datasets considered. Furthermore, they look very similar among distinct integration (i.e., among Figs. S2, S3 and S4). Hence, profiles seen in $v_{1 j_{1} 1}$ that represent distinctions between AD and control in dataset 1 correspond to those seen in $v_{1 j_{2} 2}$ that represent the distinction between WT and the other three treated cell lines in dataset 2. This is a reasonable coincidence because the distinction between WT and treated cell lines is supposed to correspond to that between control and AD. Thus, our strategy can successfully relate gene expression profiles without apparent sample matching. On the other hand, if we consider $v_{1 j_{3} 3}$, the situation differs a bit. Although we expect that $v_{1 j_{3} 3}$ represent distinctions between control and two AD cell lines, they represent the distinction between AD1 and the other two cell lines: AD2 and control. This suggests the possibility that AD2 fails to represent some property of AD. Usually, this kind of investigation is impossible, because we cannot compare two gene expression profiles without sample matching. Nevertheless, our strategy enables us to figure out the discrepancy in dataset 3 if it is compared with datasets 1 and 2. Although this is just an example, detailed comparisons of $v_{\ell_{1} j_{k} k}$ between distinct $k$ (i.e. datasets) enable us to compare gene expression profiles even without sample matching.

\section{Comparisons with Previous Works}

To demonstrate the superior performance of our proposed strategy, we compared the performance to some conventional methods that integrate multiple matrices. Unfortunately, most methods have been designed for genomic science require sample matching. For example, all nine methods listed in reference ${ }^{15}$ require sample matching. intNMF ${ }^{16}$ is also limited to datasets attributed to the same individuals. In contrast to these studies, although a review ${ }^{17}$ comprehensively reported on integrated multi-view analyses, samples were implicitly assumed to be shared, and no integrated methods aimed to integrate multiple matrices or tensors that did not share samples; only features were included. Although MINT ${ }^{18}$ was proposed to integrate gene expression profiles sharing genes rather than samples, the method assumed common labeling among multiple studies in a supervised learning framework. On the other hand, its unsupervised framework required the common quantitative variables associated with all samples included in the studies; thus, this method cannot be applied to the present task of investigating the correlations between latent variables and classifications not shared between individual studies, as shown in Table S2. We could not find any implementations designed to integrate multiple gene expression profiles formatted as matrices or tensors sharing only genes and not samples. Thus, we were forced to seek suitable methods outside the genomic field. First, we applied CMF ${ }^{19}$, implemented as a CMF package in $\mathrm{R}^{20}$, to the integration of datasets 1,2 , and 3. We computed four latent variables assuming a Poisson distribution for gene expression. None of the obtained latent variables were significantly correlated with classification in datasets 1, 2, and 3 (Table S9). Then, we tried GFA ${ }^{21}$, which was also implemented as a GFA package in R. Although we computed five latent variables, none were correlated with the classification in dataset 1 , although some were correlated with the classifications in datasets 2 and 3 (Table S9). Hence, these two advanced methods, CMF and GFA, failed to identify latent variables correlated with classifications of all three datasets using integrated analysis. Finally, we tried simple concatenation. Ironically, SVD applied to a $60617 \times 40$ contracted matrix comprising $x_{i j_{1}} \in \mathbb{R}^{60617 \times 9}, x_{i j_{2}} \in \mathbb{R}^{60617 \times 23}$ and $x_{i j_{3}} \in \mathbb{R}^{60617 \times 8}$ gave us the first singular value vector, which was correlated with all classifications of the three datasets. However, the $P$-values for the correlation with classification of dataset 1 were only very slightly significant $(P=0.05$, Table S9). We then selected 147 genes using Eq. (9) by replacing $u_{\ell_{1} i}$ with the first singular-value vectors correlated with all classifications of datasets 1 , 2 , and 3. To confirm the inferiority of simple concatenations toward TD-based unsupervised FE, although we evaluated their 
enrichment by uploading 147 genes to Enrichr, DAVID, and g:Profiler, their enrichments were clearly poorer than those of genes selected by TD-based unsupervised FE (Table S2(D) and Data S5). Thus, we could not find any other methods comparable with or superior to TD-based unsupervised FE. Considering that the two advanced methods, CMF and GFA, were inferior to the SVD applied to simple concatenated matrices, the methods developed for this purpose (i.e., integrated analysis of multiple matrices) seem to be inapplicable to the present purpose. Hence, more advanced methods must be developed to be suitable for the purpose of the present work (i.e., integrated analysis of gene expression profiles that lack common matching samples).

\section{Treatment of Missing Values}

An additional advantage of the proposed strategy may be noted as a by-product. scRNA-seq data is known to include many missing values, and matrix representation was previously employed to resolve this problem ${ }^{22}$. In the proposed strategy, although the employment of SVD and HOSVD did not aim to fill missing values, it should naturally function as a mechanism to do so. This may be the reason the present strategy also works for scRNA-seq data as well with neither any specific additional modifications nor implementations toward the treatment of scRNA-seq data set. Notably our proposed strategy successfully provided a platform to integrate typical gene expression profile measurements with small samples using scRNA-seq, which includes massive numbers of single cells, because both can be represented as a tensor form, $x_{i j k} \in \mathbb{R}^{N \times L \times K}$, no matter how large the number of cells included in the scRNA-seq. Because we can process scRNA-seq with $L=10$ in this study, this might suggest the possibility that scRNA-seq does not provide the expected large amount of information according to the number of single cells.

\section{Conclusion}

In this study, we proposed a new strategy that integrates multiple gene expression profiles that lack both sample matching and common labeling. This strategy successfully integrated $\mathrm{AD}$ gene expression profiles that lacked sample matching and $\mathrm{AD}$ scRNA-seq datasets. The former can be used for drug repositioning and TL. Massive amounts of computational memory can be saved with the latter. The proposed strategy appears to be useful for integrating gene expression profiles, even those lacking both sample matching and common labeling among them.

\section{Methods}

\section{Mathematical Formulations}

\section{Integration of multiple gene expression profiles with TD}

Here, we consider cases in which the number of genes, $N$, is much larger than the number of samples in the $k$ th gene expression profile, $M_{k}$, as $N \gg M_{k}$. Given $K,(1 \leq k \leq K)$ gene expression profiles represented as a matrix,

$$
x_{i j_{k}} \in \mathbb{R}^{N \times M_{k}}
$$

or a $(S+1)$-mode tensor,

$$
x_{i j_{k}[1] j_{k}^{[2]} \ldots j_{k}^{[S]}} \in \mathbb{R}^{N \times M_{k}^{[1]} \times M_{k}^{[2]} \times \cdots \times M_{k}^{[S]},}
$$

where $1 \leq s \leq S$ stands for the sth experimental condition in the $k$ th gene expression profile. Then, singular value decomposition (SVD) or higher-order SVD $\left(\right.$ HOSVD $\left.^{5}\right)$ is applied to obtain

$$
x_{i j_{k}}=\sum_{\ell} u_{\ell i}^{[k]} \lambda_{\ell}^{[k]} v_{\ell, j_{k}}^{[k]}
$$

where $u_{\ell i}^{[k]} \in \mathbb{R}^{N \times M}$ are eigenvectors attributed to genes . $u_{\ell j}^{[k]} \in \mathbb{R}^{M \times M}$ are eigenvectors attributed to samples, $\lambda_{\ell}^{[k]}$ are eigenvalues, or

$$
\begin{aligned}
x_{i j_{k}^{[1]} j_{k}^{[2]} \ldots j_{k}^{[S]}} & =\sum_{\ell_{1} \ell_{2} \cdots \ell_{S} \ell_{S+1}} G\left(\ell_{1} \ell_{2} \cdots \ell_{S} \ell_{S+1}\right) \\
& \times u_{\ell_{1} j_{k}^{[k]} \ell_{2} j_{k}^{[2]}}^{[k]} \cdots u_{\ell_{S} j_{k}^{[k]}}^{[k]} u_{\ell_{S+1} i}^{[k]}
\end{aligned}
$$

where $G\left(\ell_{1} \ell_{2} \cdots \ell_{S} \ell_{S+1}\right) \in \mathbb{R}^{M_{k}^{[1]} \times M_{k}^{[2]} \times \cdots \times M_{k}^{[S]} \times N}$ is a core tensor representing a weight of products, $u_{\ell_{1} j_{k}^{[1]}}^{[k]} u_{\ell_{2} j_{k}^{[2]}}^{[k]} \cdots u_{\ell_{S} j_{k}^{[S]}}^{[k]} u_{\ell_{S+1}}^{[k]}$, $u_{\ell_{s} j_{k}^{[s]}}^{[k]} \in \mathbb{R}^{M_{k}^{[s]} \times M_{k}^{[s]}}$, and $u_{\ell_{S+1} i}^{[k]} \in \mathbb{R}^{N \times N}$ are singular value orthogonal matrices. Thus, we derive the reduced matrices as either

$$
x_{i \ell k}=\sum_{j_{k}=1}^{M_{k}} x_{i j_{k}} v_{\ell j_{k}}^{[k]}, 1 \leq \ell \leq L,
$$


or

$$
\begin{aligned}
& x_{i \ell k}=\sum_{j_{k}^{[1]}=1}^{M_{k}^{[1]}} \cdots \sum_{j_{k}^{[S]}=1}^{M_{k}^{[S]}} x_{i j_{k}^{[1]} j_{k}^{[2]} \cdots j_{k}^{[S]}} u_{\ell_{1} j_{k}^{[k]}}^{[k]} \cdots u_{\ell_{S} j_{k}^{[S]}}^{[k]}, \\
& 1 \leq \ell_{s} \leq L_{k}^{[s]}\left(\leq M_{k}^{[s]}\right), 1 \leq \ell \leq L=\prod_{s=1}^{S} L_{k}^{[s]} .
\end{aligned}
$$

This implementation involves a problem of note. For example, in Eq. (4), simultaneous changes, $u_{\ell i}^{[k]} \rightarrow-u_{\ell i}^{[k]}$ and $v_{\ell j_{k}}^{[k]} \rightarrow-v_{\ell j_{k}}^{[k]}$, also satisfy Eq. (4). Hence, we cannot fix the signs of $u_{\ell i}^{[k]}$ and $v_{\ell j_{k}}^{[k]}$. This does not present a problem if we are only interested in individual gene expression profiles. However, if we need to compare multiple profiles, it does. For example, we compare multiple profiles in single-cell RNA-sequence (scRNA-seq) data analysis in this study. Thus, we fix signs of $v_{\ell j_{k}}^{[k}$ such that the correlation coefficients between $u_{\ell i}^{[1]}$ and $u_{\ell i}^{[k]}, k>1$ have positive values prior to the computations in Eq. (6).

HOSVD is applied to $x_{i \ell k} \in \mathbb{R}^{N \times L \times K}$ to obtain

$$
x_{i \ell k}=\sum_{\ell_{1}=1}^{N} \sum_{\ell_{2}=1}^{L} \sum_{\ell_{3}=1}^{K} G\left(\ell_{1} \ell_{2} \ell_{3}\right) u_{\ell_{1} i} u_{\ell_{2} \ell} u_{\ell_{3} k}
$$

where $G\left(\ell_{1} \ell_{2} \ell_{3}\right) \in \mathbb{R}^{N \times L \times K}$ is a core tensor, and $u_{\ell_{1} i} \in \mathbb{R}^{N \times N} . u_{\ell_{2} \ell} \in \mathbb{R}^{L \times L}$ and $u_{\ell_{3} k} \in \mathbb{R}^{K \times K}$ are singular value matrices that are orthogonal.

\section{Gene selection}

We can also select genes, $i$, by attributing $P$-values to the $i$ th genes while assuming $u_{\ell_{1} i}$ obeys a Gaussian distribution,

$$
P_{i}=P_{\chi^{2}}\left[>\sum_{\ell_{1} \in \Omega_{\ell_{1}}}\left(\frac{u_{\ell_{1} i}}{\sigma_{\ell_{1}}}\right)^{2}\right],
$$

where $P_{\chi^{2}}[>x]$ is the cumulative $\chi^{2}$ distribution where the argument is larger than $x, \sigma_{\ell_{1}}$ is the standard deviation, and the summation of $\ell_{1}$ is taken over a set of $\ell_{1}, \Omega_{\ell_{1}}$, which is a set of $\ell_{1}$ selected as having larger $\sum_{\ell, \ell_{3}} G\left(\ell_{1} \ell \ell_{3}\right)^{2}$. $P_{i}$ are corrected via the Benjamini-Hochberg $(\mathrm{BH})$ criterion $^{5}$, and $i$ is associated with adjusted $P$-values less than 0.01 .

\section{Projection of individual gene expression profiles onto the space spanned by $u_{\ell_{1} i}$}

To see how samples $j_{k}$ within each $K$ gene expression profile are located in the space spanned by the obtained singular value vectors attributed to genes, $u_{\ell_{1} i}$, we project individual gene expression profiles onto the space spanned by $u_{\ell_{1} i}$ as

$$
v_{\ell_{1} j_{k} k}=\sum_{i=1}^{N} u_{\ell_{1} i} x_{i j_{k} k}
$$

or

$$
v_{\ell_{1} j_{k}^{[1]} j_{k}^{[2]} \ldots j_{k}^{[S]} k}=\sum_{i=1}^{N} u_{\ell_{1} i} x_{i j_{k}^{[1]} j_{k}^{[2]} \ldots j_{k}^{[S]}},
$$

where $v_{\ell_{1} j_{k} k} \in \mathbb{R}^{N \times M_{k} \times K}$ and $v_{\ell_{1} j_{k}^{[1]} j_{k}^{[2]} \ldots j_{k}^{[S]}} \in \mathbb{R}^{N \times M_{1} \times \cdots \times M_{S} \times K}$ are regarded as coordinates of individual samples, $j_{k}$ or $j_{k}^{[s]}$, in the space spanned by $u_{\ell_{1} i}$.

\section{Gene Expression Profiles}

As denoted in the introduction, we specifically focus on Alzheimer's disease (AD) in this study. All gene expression profiles included in the six studies listed below are selected from the gene expression omnibus (GEO). Individual profiles are normalized to have zero mean and a standard deviation of one within individual profiles. Thus, when gene expression profiles are formatted as a matrix, $x_{i j_{k}}, \in \mathbb{R}^{N \times M_{k}}$ representing expression of the $i$ th gene at the $j_{k}$ th sample,

$$
\begin{aligned}
& \sum_{i=1}^{N} x_{i j_{k}}=0, \\
& \sum_{i=1}^{N} x_{i j_{k}}^{2}=N,
\end{aligned}
$$


and when gene expression profiles are formatted as a tensor, $x_{i j_{k}^{[1]} \ldots j_{k}^{[S]}} \in \mathbb{R}^{N \times M_{k}^{[1]} \times \cdots \times M_{k}^{[S]}}$, representing the expression of the $i$ th gene at the sample annotated by indices $j_{1}, \cdots, j_{S}$, and

$$
\begin{aligned}
& \sum_{i=1}^{N} x_{i j j_{k}^{[1] \ldots j}}=0 \\
& \sum_{i=1}^{N} x_{i j_{k}^{[1]} \ldots j_{k}^{[S]}}^{2}=N .
\end{aligned}
$$

\section{Dataset 1: GSE160224}

The datase ${ }^{23}$ denoted as dataset 1 (i.e., $k=1$ ) in this study comprises as few as nine samples, including on-demented controls, three APP duplications, and three isogenically corrected induced pluripotent stem-cell lines. In this study, three control samples are treated as controls, and the other six are treated samples when the coincidence to singular value vectors or latent variables are investigated, and genes whose expression are altered between these two classes are intended to be selected. Gene expression profiles are measured by RNA-seq technology, and genes are annotated using the Ensembl gene ID. The genes whose expressions are measured numbers as many as 58,302 in contrast to the small number of samples. As a result, gene expression profiles are formatted as a matrix, $x_{i j_{1}} \in \mathbb{R}^{58302 \times 9}$, representing expressions of the $i$ th gene of the $j_{1}$ th sample.

\section{Dataset 2: GSE155567}

The datase ${ }^{24}$ denoted as dataset 2 (i.e., $k=2$ ) in this study comprises four classes, including THP1 macrophages after the knockout of CD33 and/or the knockdown (silencing) of PTPN6 (six samples per class). Thus, in all, it includes as few as 24 samples. In this study, we do not specifically assume which classes are considered controls, but they are regarded as four categorical classes when the coincidence with singular value vectors or latent variables are investigated. For unknown reasons, although gene expression profiles of one sample are missing, it is unlikely to affect the outcome because it still includes most (23 out of 24) samples. Gene expression profiles are measured by RNA-seq technology, and genes are annotated using the Ensembl gene ID. The number of genes whose expressions are measured amount to as many as 60,617 in contrast to the small number of samples. As a result, gene expression profiles are formatted as a matrix, $x_{i j_{2}} \in \mathbb{R}^{60617 \times 23}$, representing the expression of the $i$ th gene of the $j_{2}$ th sample.

\section{Dataset 3: GSE162873}

The dataset ${ }^{25}$ denoted as dataset 3 (i.e., $k=3$ ) in this study comprises eight samples, four of which are AD cell lines, and the other four are normal. Because four $\mathrm{AD}$ cell lines comprise two sets of two samples using two distinct cell lines, this dataset includes three categorical classes when the coincidence with singular value vectors or latent variables is investigated. Two samples are taken from the first AD cell line, two samples are taken from the second AD cell line, and four samples are taken from normal cell lines. Gene expression profiles are measured by RNA-seq technology, and genes are annotated using the Ensembl gene ID. The number of genes whose expressions are measured amounts to as many as 47,749 in contrast to the small number of samples. As a result, gene expression profiles are formatted as a matrix, $x_{i j_{3}} \in \mathbb{R}^{47749 \times 8}$, representing the expression of the $i$ th gene of the $j_{3}$ th sample.

\section{Dataset 4: GSE164788}

The dataset ${ }^{11}$ denoted as dataset 4 (i.e., $k=4$ ) in this study is an in-vitro differentiated mixture of neuron and glial cells derived from the ReNcell VM neural progenitor cell line treated with 80 different compounds; mRNA levels are measured using RNA-seq. Among the 80 compounds, we select 94 drugs and combinations from which at least two doses are tested. For each dose, three biological replicates are provided. When more than three are used, we randomly select three of them. Gene expression profiles are measured by RNA-seq technology, and genes are annotated using the Ensembl gene ID. The genes whose expressions numbers as many as 28,044. As a result, gene expression profiles are formatted as a tensor, $x_{i j_{4}\left[j_{4}^{[1]} j_{4}^{[3]}\right.} \in \mathbb{R}^{28044 \times 94 \times 4 \times 3}$, representing the expression of the $i$ th gene at the $j_{1}$ th drug combination, the $j_{2}$ th dose, and the $j_{3}$ th biological replicates.

\section{Dataset 5: GSE164642}

The dataset ${ }^{26}$ denoted as dataset 5 (i.e., $k=5$ ) in this study comprises three sets of six samples; each includes two classes corresponding to ABCC1 activated cells by distinct RNA or control cells. They include three biological replicates (18 total samples). Gene expression profiles are measured by RNA-seq technology, and genes are annotated using the Ensembl gene ID. Thus, they are treated as six categorical classes when the coincidence with singular value vectors or latent variables is investigated. The genes whose expressions are measured numbers as many as 58,003 in contrast to the small number of samples. As a result, gene expression profiles are formatted as a tensor, $x_{i j j_{5}^{[1]} j_{5}^{[2]} j_{5}^{[3]}} \in \mathbb{R}^{58003 \times 3 \times 2 \times 3}$, representing the expression of the 
$i$ th gene at those treated by the $j_{5}^{[1]}$ th RNA, the $j_{5}^{[2]}$ th treatment $\left(j_{5}^{[2]}=1\right.$ :control, $j_{5}^{[2]}=2$ :ABCC1 activated, $)$ and the $j_{5}^{[3]}$ th biological replicates.

Dataset 6: GSE163577

This is a scRNA-seq dataset ${ }^{27}$ denoted as dataset 6 (i.e., $k=6$ ) in this study, including both AD and healthy controls from 25 hippocampus and superior frontal cortex samples across 17 control and eight AD patients. 25 individual datasets are formatted

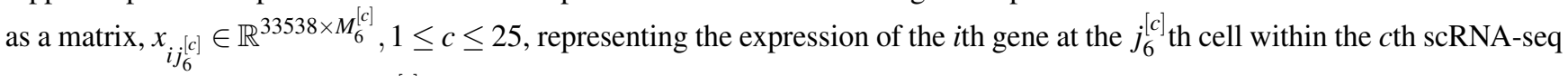
profile. The number of cells, $M_{6}^{[c]}$, in individual datasets vary and is roughly $10^{4}$.

\section{Integrated Analysis of Gene Expression Profiles}

\section{Integrated analysis of datasets 1, 2, and 3}

When we integrate the datasets 1,2, and 3, we apply SVD to them to get

$$
\begin{aligned}
& x_{i j_{1}}=\sum_{\ell} u_{\ell i}^{[1]} \lambda_{\ell}^{[1]} v_{\ell j_{1}}^{[1]} \\
& x_{i j_{2}}=\sum_{\ell} u_{\ell i}^{[2]} \lambda_{\ell}^{[2]} v_{\ell j_{2}}^{[2]} \\
& x_{i j_{3}}=\sum_{\ell} u_{\ell i}^{[3]} \lambda_{\ell}^{[3]} v_{\ell j_{3}}^{[3]}
\end{aligned}
$$

Then, we compute $x_{i \ell k}$ using Eq. (6) while setting $L=8$ because $M_{1}=9, M_{2}=24, M_{3}=8$, and $L$ cannot exceed $M_{k}$. Because the number of genes whose expressions are measured differs among datasets 1,2 , and 3, we employ $N=60617$ as the number of genes whose expressions are measured in dataset 2 and the largest number of genes measured among datasets 1, 2, and 3 . Then, these three gene expression profiles are formatted as a tensor,

$$
x_{i \ell k} \in \mathbb{R}^{60617 \times 8 \times 3},
$$

where the missing expressions in datasets 1 and 3 caused by the smaller number of genes than in dataset 2 are filled with zero. HOSVD is applied to $x_{i \ell k}$ as in Eq. (8).

\section{Drug repositioning using the tensor obtained with datasets 1, 2, and 3}

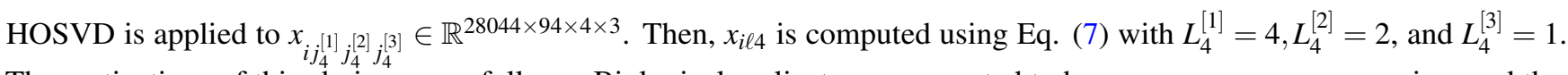
The motivations of this choice are as follows. Biological replicates are expected to have common gene expression, and the first singular value vector, $u_{1 j_{4}^{[3]]}}$, is expected to have constant value regardless of $j_{4}^{[3]}$, based upon previous studies. Thus, it is sufficient to consider only $u_{1 j_{4}^{[3]}}$ for biological replicates. Then, two choices remain, including $L_{4}^{[1]}=4, L_{4}^{[2]}=2$ or $L_{4}^{[1]}=2, L_{4}^{[2]}=4$. Clearly, the former is reasonable because the number of drug combinations, at 94 , is much larger than that of dose density, which is four. Missing values are filled with zero. In addition to $x_{i \ell k}, 1 \leq k \leq 3$, obtained in the previous subsection, HOSVD is applied to $x_{i \ell k} \in \mathbb{R}^{60617 \times 8 \times 4}$ as in Eq. (8).

\section{Transfer Learning (TL) using the tensor obtained with datasets 1, 2, and 3}

To evaluate the performance of TL, HOSVD is applied to $x_{i j} j_{5}^{[1]} j_{5}^{[2]} j_{5}^{[3]} \in \mathbb{R}^{58003 \times 3 \times 2 \times 3}$. Then, $x_{i \ell 5}$ is computed using Eq. (7) with $L_{5}^{[s]}=2,1 \leq s \leq 3$. HOSVD is applied to $x_{i \ell k} \in \mathbb{R}^{60617 \times 8 \times 4}$, obtained with Eq. (8) after $x_{i \ell 4}$ is replaced with $x_{i \ell 5}$.

\section{Integrated analysis of scRNA-seq data}

SVD is applied to $x_{i j_{6}^{[c]}}, 1 \leq c \leq 25$ one-by-one. Then, $x_{i \ell c}$ is computed by Eq. (6) while replacing $k$ with $c$ and $L=10$. Then, HOSVD is applied to $x_{i \ell c} \in \mathbb{R}^{33538 \times 10 \times 25}$.

\section{Methods to be Compared}

In the following, we tested three methods that assume the latent variables attributed to genes, $i$, which are also common among datasets 1,2 , and 3 . 


\section{Collective matrix factorization (CMF)}

To perform CMF, we used $x_{I j_{1}} \in \mathbb{R}^{60617 \times 9}, x_{i j_{2}} \in \mathbb{R}^{60617 \times 23}$, and $x_{i j_{3}} \in \mathbb{R}^{60617 \times 8}$, such that they shared the same number of genes. Missing values were filled with zero and were normalized to have zero mean and a standard deviation of one, as denoted in the beginning of this section. The structure of assumed modeling is given as

$$
x_{i j_{k}}=\sum_{\ell=1}^{L} u_{\ell i} u_{\ell j_{k}}^{[k]}+b_{i}^{[k]}+b_{j_{k}}^{[k]}+\varepsilon_{i j_{k}}^{[k]}
$$

where $u_{\ell i} \in \mathbb{R}^{L \times 60617}, u_{\ell j_{k}}^{[k]} \in \mathbb{R}^{L \times M_{k}}$, and $b_{i}^{[k]} \in \mathbb{R}^{60617}, b_{j_{k}}^{[k]} \in \mathbb{R}^{M_{k}}, \varepsilon_{i j_{k}}^{[k]} \in \mathbb{R}^{60617 \times M_{K}}$. We also employed an option wherein $x_{i j_{k}}$ obeys a Poisson distribution because the negative signed binary distribution usually assumed for the RNA-seq dataset is not provided as an option. Before applying this model to $x_{i j_{k}}$, some constants were added so that they do not take negative values, because Poisson distributions will not accept them. $L=4$ because TD-based unsupervised feature extraction (FE) can identify a singular value vector correlated with classification in datasets 1,2 , and 3 within the top four.

\section{Group factor analysis (GFA)}

The datasets used were the same as those used in the trials using CMF. The structure of assumed model is given as

$$
x_{i j_{k}}=\sum_{\ell=1}^{L} u_{\ell i} u_{\ell j_{k}}^{[k]}+\varepsilon_{i j_{k}}^{[k]}
$$

The primary difference from CMF, which employs Bayesian inferencing, is that GFA does not assume the distribution of $x_{i j_{k}}$. $L=5$ was assumed because it was employed in the example in the GFA tutorial and is larger than the number of singular value vectors computed with TD-based unsupervised FE correlated with classifications in datasets 1, 2, and 3.

\section{Simple concatenation}

A matrix, $x_{i j} \in \mathbb{R}^{60617 \times 40}$, where $40=\sum_{k=1}^{3} M_{K}$, is generated by concatenating three matrices, $x_{i j_{1}}, x_{i j_{2}}$, and $x_{i j_{3}}$, to share the row number:

$$
x_{i j}=\left\{\begin{array}{llc}
x_{i j_{1}}, & j_{1}=j, & 1 \leq j \leq 9 \\
x_{i j_{2}}, & j_{2}=j-9, & 10 \leq j \leq 32 \\
x_{i j_{3}}, & j_{3}=j-32, & 33 \leq j \leq 40
\end{array}\right.
$$

Then, SVD was applied to $x_{i j}$ to obtain

$$
x_{i j}=\sum_{\ell} u_{\ell i} \lambda_{\ell} v_{\ell j} .
$$

$v_{\ell j}$ for $1 \leq j \leq 9$ were used for the latent variables attributed to nine samples in dataset $1, v_{\ell j}$ for $10 \leq j \leq 32$ were used for the latent variables attributed to 23 samples in dataset 2 , and $v_{\ell j}$ for $33 \leq j \leq 40$ were used for the latent variables attributed to eight samples in dataset 3 .

\section{References}

1. Huang, C. et al. Integrated analysis of multiple gene expression profiling datasets revealed novel gene signatures and molecular markers in nasopharyngeal carcinoma. Cancer Epidemiol. Prev. Biomarkers 21, 166-175, DOI: 10.1158/ 1055-9965.EPI-11-0593 (2012). https://cebp.aacrjournals.org/content/21/1/166.full.pdf.

2. Hu, P. et al. Integrative analysis of multiple gene expression profiles with quality-adjusted effect size models. $B M C$ Bioinforma. 6, 128, DOI: 10.1186/1471-2105-6-128 (2005).

3. Kyoon Choi, J. et al. Integrative analysis of multiple gene expression profiles applied to liver cancer study. FEBS Lett. 565, 93-100, DOI: https://doi.org/10.1016/j.febslet.2004.03.081 (2004).

4. Yang, Z.-Y. et al. Multi-view based integrative analysis of gene expression data for identifying biomarkers. Sci. Reports $\mathbf{9}$, DOI: 10.1038/s41598-019-49967-4 (2019).

5. Taguchi, Y.-H. Unsupervised Feature Extraction Applied to Bioinformatics (Springer International Publishing, 2020).

6. Kuleshov, M. V. et al. Enrichr: a comprehensive gene set enrichment analysis web server 2016 update. Nucleic Acids Res. 44, W90-W97, DOI: 10.1093/nar/gkw377 (2016). https://academic.oup.com/nar/article-pdf/44/W1/W90/18788036/ gkw377.pdf. 
7. Huang, D. W. et al. Systematic and integrative analysis of large gene lists using DAVID bioinformatics resources. Nat. Protoc. 4, 44-57, DOI: 10.1038/nprot.2008.211 (2008).

8. Huang, D. W. et al. Bioinformatics enrichment tools: paths toward the comprehensive functional analysis of large gene lists. Nucleic Acids Res. 37, 1-13, DOI: 10.1093/nar/gkn923 (2008). https://academic.oup.com/nar/article-pdf/37/1/1/ 17059338/gkn923.pdf.

9. Raudvere, U. et al. g:Profiler: a web server for functional enrichment analysis and conversions of gene lists (2019 update). Nucleic Acids Res. 47, W191-W198, DOI: 10.1093/nar/gkz369 (2019). https://academic.oup.com/nar/article-pdf/47/W1/ W191/28879887/gkz369.pdf.

10. Taguchi, Y.-H. Identification of candidate drugs using tensor-decomposition-based unsupervised feature extraction in integrated analysis of gene expression between diseases and DrugMatrix datasets. Sci. Reports 7, DOI: 10.1038/ s41598-017-13003-0 (2017).

11. Rodriguez, S. et al. Machine learning identifies candidates for drug repurposing in alzheimer's disease. Nat. Commun. 12, DOI: $10.1038 / \mathrm{s} 41467-021-21330-0$ (2021).

12. Weiss, K. et al. A survey of transfer learning. J. Big Data 3, DOI: 10.1186/s40537-016-0043-6 (2016).

13. Chen, Y. et al. Gene expression inference with deep learning. Bioinformatics 32, 1832-1839, DOI: 10.1093/bioinformatics/ btw074 (2016).

14. ElAli, A. \& Rivest, S. The role of ABCB1 and ABCA1 in beta-amyloid clearance at the neurovascular unit in alzheimer's disease. Front. Physiol. 4, 45, DOI: 10.3389/fphys.2013.00045 (2013).

15. Cantini, L. et al. Benchmarking joint multi-omics dimensionality reduction approaches for the study of cancer. Nat. Сотmun. 12, DOI: 10.1038/s41467-020-20430-7 (2021).

16. Chalise, P. \& Fridley, B. L. Integrative clustering of multi-level 'omic data based on non-negative matrix factorization algorithm. PLOS ONE 12, 1-18, DOI: 10.1371/journal.pone.0176278 (2017).

17. Li, Y. et al. A review on machine learning principles for multi-view biological data integration. Briefings Bioinforma. 19, 325-340, DOI: 10.1093/bib/bbw113 (2016). https://academic.oup.com/bib/article-pdf/19/2/325/25524236/bbw113.pdf.

18. Rohart, F. et al. MINT: a multivariate integrative method to identify reproducible molecular signatures across independent experiments and platforms. BMC Bioinforma. 18, DOI: 10.1186/s12859-017-1553-8 (2017).

19. Klami, A. et al. Group-sparse embeddings in collective matrix factorization (2014). 1312.5921.

20. R Core Team. R: A Language and Environment for Statistical Computing. R Foundation for Statistical Computing, Vienna, Austria (2020).

21. Leppäaho, E. et al. GFA: Exploratory analysis of multiple data sources with group factor analysis. J. Mach. Learn. Res. 18, $1-5$ (2017).

22. Hu, Y. et al. WEDGE: imputation of gene expression values from single-cell RNA-seq datasets using biased matrix decomposition. Briefings Bioinforma. DOI: 10.1093/bib/bbab085 (2021). Bbab085, https://academic.oup.com/bib/ advance-article-pdf/doi/10.1093/bib/bbab085/36992506/bbab085.pdf.

23. Ye, T. et al. Efficient manipulation of gene dosage in human iPSCs using CRISPR/cas9 nickases. Commun. Biol. 4, DOI: 10.1038/s42003-021-01722-0 (2021).

24. Wißfeld, J. et al. Deletion of Alzheimer's disease-associated CD33 results in an inflammatory human microglia phenotype. Glia 69, 1393-1412, DOI: 10.1002/glia.23968 (2021).

25. Hanna, R. et al. G-quadruplexes originating from evolutionary conserved 11 elements interfere with neuronal gene expression in Alzheimer's disease. Nat. Commun. 12, DOI: 10.1038/s41467-021-22129-9 (2021).

26. Jepsen, W. M. et al. Adenosine triphosphate binding cassette subfamily c member 1 (ABCC1) overexpression reduces APP processing and increases alpha- versus beta-secretase activity, in vitro. Biol. Open DOI: 10.1242/bio.054627 (2020).

27. Yang, A. C. et al. A human brain vascular atlas reveals diverse cell mediators of Alzheimer's disease risk. bioRxiv DOI: 10.1101/2021.04.26.441262 (2021). https://www.biorxiv.org/content/early/2021/04/27/2021.04.26.441262.full.pdf.

\section{Acknowledgements}

This work was supported by KAKENHI [grant numbers 19H05270, 20H04848, and 20K12067] to YHT. 


\section{Author contributions statement}

YHT planned the research and performed analyses. YHT and TT evaluated the results, discussions, and outcomes and wrote and reviewed the manuscript.

\section{Additional information}

Supplementary Tables (Tables S1 to S9) and supplementary Figures (Figs. S1 to S5). Supplementary Data S1 to S5. Source.R and sample.csv: Sample source code and a file required to execute a sample source code. 


\section{Supplementary Files}

This is a list of supplementary files associated with this preprint. Click to download.

- DataS1.xlsx

- DataS2.xlsx

- DataS3.xlsx

- DataS4.xlsx

- DataS5.xlsx

- sample.csv

- SupplementaryTables.pdf

- SupplementaryFigures.pdf

- source.r 